illustrates, by setting up a complete system of invariants and covariants for the general system of differential equations $S$ of the theory of curved surfaces, a fact of which he has made use in previous papers (see abstracts in the January BuLlETIN, pages 171,172$)$. The invariants and covariants calculated for the system $S^{\prime}$ may be expressed explicitly in terms of the coefficients of $S$, without actually requiring the integration necessary to throw $S$ into the form $S^{\prime}$. Not only is this procedure preferable on account of the simplifications which result from the use of the canonical form $S^{\prime}$, but it also gives an insight into the real nature of the invariants and covariants.

34. In a recent paper* Professor L. E. Dickson has discussed the algebra defined by the relations

$$
x y=y \theta(x), \quad y^{n}=g,
$$

where $\theta(x)$ is a rational polynomial in $x$ in a given field $F$, and $y^{n}$ is the first power of $y$ which is commutative with $x$. In this note Professor Wedderburn gives a simple proof that, if $g$ is properly chosen in $F$, this algebra is primitive, i. e., division by any number of the algebra except zero is always possible.

$$
\begin{aligned}
& \text { F. N. Cole, } \\
& \text { Secretary. }
\end{aligned}
$$

\title{
WINTER MEETING OF THE SOCIETY AT CHICAGO.
}

The thirty-second regular meeting of the Chicago Section of the American Mathematical Society was held at the University of Chicago on Friday and Saturday, December 26-27, 1913. In accordance with a recent action of the Council the meetings of the Chicago Section for the presentation of scientific papers are hereafter to be designated as meetings of the Society at Chicago, and this was the first occasion to be observed under the new arrangement. Seventy-three persons were in attendance, including the following fifty-six members of the Society:

Professor W. H. Bates, Professor G. A. Bliss, Dr. Henry Blumberg, Professor Daniel Buchanan, Professor W. H. Butts, Professor H. E. Cobb, Professor D. R. Curtiss, Professor S. C. Davisson, Professor L. E. Dickson, Professor Arnold Dresden,

*Transactions, Jan., 1914. 
Professor Arnold Emch, Professor Peter Field, Professor W. B. Ford, Dr. Tomlinson Fort, Professor A. B. Frizell, Mr. M. G. Gaba, Professor A. G. Hall, Professor E. R. Hedrick, Dr. T. H. Hildebrandt, Professor T. F. Holgate, Mr. L. A. Hopkins, Professor L. C. Karpinski, Professor A. M. Kenyon, Professor C. E. Love, Professor A. C. Lunn, Professor W. D. MacMillan, Dr. H. F. MacNeish, Professor C. R. Mann, Professor J. L. Markley, Professor G. A. Miller, Professor E. H. Moore, Professor E. J. Moulton, Professor F. R. Moulton, Professor G. W. Myers, Mr. V. C. Poor, Professor H. L. Rietz, Dr. Mildred Sanderson, Miss Ida M. Schottenfels, Mr. A. R. Schweitzer, Professor J. B. Shaw, Dr. H. M. Sheffer, Professor C. H. Sisam, Professor H. E. Slaught, Professor A. W. Smith, Mr. R. B. Stone, Professor A. L. Underhill, Professor J. N. Van der Vries, Professor E. B. Van Vleck, Miss Mary E. Wells, Professor Marion B. White, Professor E. J. Wilczynski, Professor F. B. Wiley, Dr. K. P. Williams, Professor A. E. Young, Professor J. W. A. Young, Professor Alexander Ziwet.

The reading of papers occupied four half-day sessions. Professor E. B. Van Vleck, President of the Society, occupied the chair on Saturday morning, and Professor Alexander Ziwet, past Vice-President, on Friday afternoon during the presentation of the retiring address by Professor D. R. Curtiss, chairman of the Chicago Section. Professor Curtiss presided during the rest of Friday afternoon and on Friday morning, and Professor E. J. Wilczynski, the newly elected chairman, on Saturday afternoon.

At the business meeting on Saturday morning the following officers of the Chicago Section were elected: Chairman, Professor E. J. Wilczynski; Secretary, Professor H. E. Slaught; and third member of the program committee, Professor A. M. Kenyon. The presentation of a formal paper by the retiring chairman has proved so satisfactory that the program committee was requested to arrange for more papers of this character; and since the chairmen now serve two years it was proposed to invite past chairmen to present such papers in the alternate years. Various suggestions were also referred to the committee for relieving the programs of their present congestion, particularly the plan of dividing the papers into groups and holding two simultaneous sessions during part of the meeting. 
On Friday evening the University of Chicago tendered a complimentary dinner to the members of the Society and others attending the meeting. President Van Vleck presided on this occasion and Dean James R. Angell represented the University in the absence of President Judson from the city. There were informal addresses on the growth and influence of the American Mathematical Society from its inception in 1894, particularly with reference to the contribution of the West since the organization of the Chicago Section in 1896, by President Van Vleck, Professor Moore, first chairman of the Section, Professor Holgate, first secretary, and Professor Slaught, present secretary. These were followed by an address of welcome and congratulation combined in felicitous strain by Dean Angell. President Van Vleck expressed the gratitude of the Society for the cordial hospitality of the University of Chicago, and at the subsequent business meeting on Saturday morning he presented a formal resolution of thanks, which was adopted and transmitted to President Judson.

The following papers were presented at this meeting:

(1) Professor ARnold Eмch: "On the properties of certain systems of functions."

(2) Professor L. C. Karpinski: "The algebra of Rollandus."

(3) Professor W. D. MacMillan: "A reduction of certain analytic differential equations to differential equations of an algebraic type."

(4) Professor W. D. MacMillan: "On Foucault's pendulum."

(5) Miss Olive Hazlett: "Invariants which characterize some linear associative algebras."

(6) Mr. A. R. SchweItzer: "Note on functional equations."

(7) Professor Daniel Buchanan: "Periodic orbits on a smooth surface."

(8) Professor Daniel Buchanan: "Periodic motion in a curve approximating a vertical parabola."

(9) Professor D. R. Cuntiss: "Laguerre's extension of Descartes' rule of signs."

An interval was here devoted to informal questions and discussion.

(10) Mr. Vincent C. Poon: "Some exact solutions of differential equations of motion of an incompressible viscous fluid." 
(11) Professor G. A. Miller: "A non-abelian group whose group of isomorphisms is abelian."

(12) Professor G. A. Mirler: "A group of order $p^{m}$ whose group of isomorphisms is of order $p^{a}$."

(13) Professor G. A. Miller: "A group admitting outer isomorphisms which do not permute any sets of conjugate operators."

(14) Professor F. R. Moulton: "On the deviations of falling bodies."

(15) Professor E. J. Wilczynski: "A new representation for a certain class of surfaces with indeterminate directrix curves."

(16) Professor C. E. Love: "On the irregular integrals of linear differential equations."

(17) Professor J. B. Shaw: "The two fundamental operations of general vector analysis."

(18) Professors G. A. Bliss and A. L. Underhill: "The minimum of a definite integral for unilateral variations in space."

(19) Professor G. A. Buiss: "On the Weierstrass Efunction."

(20) Dr. Henry Blumberg: "A general theorem regarding sets of first and of second category."

(21) Dr. Henry Blumberg: "The oscillation equation $\omega \omega f(x)=g(x) \omega f(x) . "$

(22) Professor A. B. Frizell: "A non-enumerable wellordered set."

(23) Professor E. J. Moulton: "On figures of equilibrium of a rotating compressible fluid body."

(24) Professor L. E. Dickson: "A linear associative division algebra in $n^{2}$ units."

(25) Professor L. E. Dickson: "A form of Cayley's algebra from which its remarkable properties may be deduced at once."

(26) Mr. A. R. Schweitzer: "On quasi-transitive and symmetric functions."

(27) Mr. A. R. SchweItzer: "On the sensed and non-sensed point fields in the foundations of geometry."

(28) Professor Peter Field: "On constrained motion."

(29) Dr. K. P. Williams: "Concerning the linear difference equations of first order."

(30) Dr. C T. Sullivan: "Concerning a certain set of organic curves on a ruled surface." 
(31) Dr. G. E. Wahlin: "The binomial equation of prime degree in an arbitrary domain."

(32) Professor G. N. Bauer and Dr. H. L. Slobin: "Algebraic and transcendental numbers."

(33) Professor G. N. BAUER: "Conditionally convergent double series."

(34) Professor W. H. Bates: "The curvatures of a $\lambda$-space $R_{\lambda}$ represented as differential parameters of an $n$-space containing $R_{\lambda}$."

Miss Hazlett was introduced by Professor Dickson. The papers of Mr. Schweitzer, Dr. Wahlin, Dr. Sullivan, Professor Bauer, and the second and third papers of Professor Miller were read by title.

Under the head of informal questions and discussions, the Secretary presented some data concerning the membership of the Society in which it appeared that of the approximately 600 colleges listed by the United States Commissioner of Education only about 150 are represented in the Society by members of their faculties; and the question was raised whether the Society's influence ought not to embrace at least all those institutions in which courses in collegiate mathematics, including the calculus, are taught.

Abstracts of the formal papers are given below, the numbers corresponding to those in the list of titles above.

1. In the theory of implicit real functions of several variables the cases considered are mostly those in which the corresponding Jacobians do not vanish simultaneously for a set of variables within the interval for which the functions are defined. On the other hand, when this happens, we may expect particular relations between the functions which deserve to be investigated. It is the purpose of Professor Emch's paper to show the importance of such cases by studying a certain system of functions and their Jacobians in connection with a geometrical problem. The paper will appear in the Annals of Mathematics.

2. In 1424 Rollandus, canon of St. Chapelle in Paris, wrote at the command of John, Duke of Lancaster, a summary of mathematics. This important work is preserved in manuscript, in a unique copy so far as we know, in the library of Mr. G. A. Plimpton. The treatise includes a discussion on 
algebra which is found in the second section of the second part of the work and extends from folio $84^{\prime}$ to $110^{\prime}$. Professor Karpinski continues his series of studies on medieval algebra with an examination of this work. The study shows that Rollandus drew very extensively from the Quadripartitum Numerorum of Johannes de Muris whose work in algebra has been analyzed in an earlier paper of this series.* In many parts the work is largely a copy of the treatise which preceded it by a century. This mathematical treatise of Rollandus is of importance not only because it shows the influence of de Muris in France, but more particularly because it throws valuable light on the state of mathematical learning in France in the early fifteenth century.

3. Suppose that there is given a set of differential equations

$$
\frac{d x_{i}}{d t}=X_{i}\left(x_{1}, \cdots, x_{n}\right) \quad(i=1, \cdots, n),
$$

and that $X_{i}(0, \cdots, 0)=0$; suppose further that the $X_{i}$ functions are expansible in powers of the $x_{j}$, and that the $x_{j}$ functions are normal variables, so that the equations can be written

$\frac{d x_{i}}{d t}=\alpha_{i} x_{i}+\sum_{j_{1}=0}^{\infty} \cdots \sum_{j_{n}=0}^{\infty} \beta_{j_{1}}^{(i)} \cdots j_{n} x_{1}{ }^{j_{1}} x_{2}{ }^{j_{2}} \cdots x_{n}{ }^{j_{n}} \quad\left\{\begin{array}{c}i=1, \cdots, n \\ \sum_{k=1}^{k=n} j_{k} \geqq 2\end{array}\right\}$

then the question raised by Professor MacMillan is this: Can there be found a convergent substitution

$x_{i}=y_{i}+\sum_{j_{1}=0}^{\infty} \cdots \sum_{j_{n}=0}^{\infty} \gamma_{j 1}^{(i)} \ldots j_{n} y_{1}^{j_{1}} \cdots y_{n}^{j_{n}} \quad\left\{\begin{array}{c}i=1, \cdots, n \\ \Sigma_{k=1}^{k=n} j_{k} \geqq 2\end{array}\right\}$

such that the resulting differential equations in the variables $y_{i}$

$$
\frac{d y_{i}}{d t}=Y_{i}\left(y_{1}, \cdots, y_{n}\right) \quad(i=1, \cdots, n)
$$

are algebraic, and in particular that the $Y_{i}$ are polynomials in $y_{1}, \cdots, y_{n}$. In answer to the question it is shown that, if the constants $\alpha_{i}$ satisfy a very simple condition, such a substitution can be found for which the resulting differential equations are

* Bibliotheca mathematica, ser. 3, vol. 13, pp. 99-114 (May, 1913). 


$$
\frac{d y_{i}}{d t}=\alpha_{i} y_{i} \quad(i=1, \cdots, n)
$$

In case the condition is not satisfied by all of the $\alpha_{i}$ but is satisfied by a group of them, say $\alpha_{1}, \cdots, \alpha_{k}$, then there exists a substitution

$$
\begin{aligned}
& x_{i}=y_{i}+\sum_{j_{1}=0}^{\infty} \cdots \sum_{j_{k}=0}^{\infty} \gamma_{j_{1}}^{(i)} \cdots j_{k} y_{1}{ }^{j_{1}} \cdots y_{k}^{j_{k}} \quad(i=1, \cdots, k), \\
& x_{i}=0+\sum_{j_{1}=0}^{\infty} \cdots \sum_{j_{k}=0}^{\infty} \gamma_{j_{1}}^{(i)} \cdots j_{k} y_{1}^{j_{1}} \cdots y_{k}^{j_{k}} \quad(i=k+1, \cdots, n),
\end{aligned}
$$

for which the resulting differential equations are

$$
\frac{d y_{i}}{d t}=\alpha_{i} y_{i} \quad(i=1, \cdots, k) .
$$

In the first case the transformed equations are equivalent to the original within the region of convergence of the substitution, for there is a unique correspondence between the values of the $x_{i}$ and $y_{i}$ in this region. In the second case the number of variables $y_{i}$ is less than the number of the $x_{i}$ so that such a complete correspondence is not possible. The cases where the above conditions are not satisfied lead to an extended field which has as yet been but slightly investigated by the writer.

4. No rigorous solution of the equations of motion of Foucault's pendulum has hitherto been given. In this paper by Professor MacMillan a rigorous solution is obtained by the methods of periodic orbits. For a given pendulum there are two arbitrary constants, namely, the amplitude of the oscillation and the azimuth of the initial plane of motion. In the present solution the amplitude of the oscillation is arbitrary, but the initial plane of motion is either the plane of the meridian or the prime vertical.

5. In this paper Miss Hazlett finds invariants which completely characterize linear associative algebras of two and three units with constants of multiplication and coordinates in the field $C$ of all real and complex numbers. By invariant is here meant a single-valued function (in Dirichlet's sense of correspondence) of the constants of multiplication which takes the same value for two algebras of the same number of units and 
having constants of multiplication and coordinates in any field $F$, if the two algebras be equivalent with respect to $F$. The invariants $I_{1}, \cdots, I_{m}$ are said to characterize completely the algebras of $n$ units over a field $F$ when each $I_{k}$ has the same value for two algebras only when they are equivalent with respect to $F$.

For any field $F$ the rational integral invariants and covariants of the characteristic equations and of the coefficients of the powers of $\omega$ therein (regarded as functions of the coordinates of the general number of the algebra) are invariants and covariants respectively of the linear algebra; and they are expressible as rational integral functions of the determinants and minors of the $2 n^{2}$ linear equations which are necessary and sufficient conditions for the existence of a modulus.

In view of this, from Study's results in the Göttinger Nachrichten for 1889 it follows that, in the field $C$, the ternary linear associative algebras with a modulus are completely characterized by their rank and by the rank of the discriminant of the coefficient of $\omega$ in either characteristic equation. By a suitable reduction, it is clear that the binary linear associative algebras (with or without a modulus) are completely characterized by the identical vanishing or non-vanishing of the coefficient of $\omega$ and by the rank of the discriminant of the coefficient of $\omega^{0}$ in either characteristic equation.

6. Mr. Schweitzer proves the following theorem: If

and

$$
\begin{gathered}
\phi\{x, \phi(y, z)\}=\phi\{z, \phi(y, x)\}, \\
\phi\{f(x, y), y\}=\psi(x), \quad f\{\phi(x, y), x\}=\psi(y),
\end{gathered}
$$

$$
\phi\{\psi(x), y\}=\psi \phi(x, y),
$$

then there exists a function $\chi(x)$ such that

$$
\chi[\phi(x, y)]=\chi(x)+\chi(y), \quad \chi[\psi(x)]=\chi(x)+c
$$

and

$$
\chi[f(x, y)]=\chi(x)-\chi(y)+c,
$$

where $c$ is a constant.

7. In his first paper Professor Buchanan proves the existence, and gives a practical method for the construction, of 
periodic orbits described by a particle which moves, subject to gravity, on a smooth surface. When the surface is a sphere the problem reduces to the well known problem of the spherical pendulum. Taking the general solutions of the spherical pendulum as derived in Chapter III of Professor Moulton's Periodic Orbits, which will appear shortly, Professor Buchanan determines periodic orbits described on the surface $S+\epsilon S^{\prime}=0$, where $S=0$ is the equation of the sphere in the problem of the spherical pendulum, $S^{\prime}$ is an analytic function of the three rectangular coordinates, and $\epsilon$ is a variable parameter. The solutions are expansible as power series in $\epsilon$ in which the coefficients are trigonometric functions of the time; and the period is the same as that of the vertical motion of the spherical pendulum.

The equation of the surface employed in this paper is the same as that used by Poincaré in his memoir "Sur les lignes géodésiques des surfaces convexes.”* Periodic orbits play the same rôle in this paper as closed geodesics in Poincaré's memoir.

8. Using the same method as in his first paper, Professor Buchanan determines a periodic solution for the motion of a particle which moves, subject to gravity, in the curve

$$
x^{2}-p y+\epsilon f(x)=0,
$$

where $\epsilon$ is a parameter and $f(x)$ is analytic in $x$. For $\epsilon=0$ the curve becomes a vertical parabola and a periodic solution can be derived by means of elliptic integrals. Then the analytic continuation of this solution is made with respect to $\epsilon$ as in the first paper.

9. The paper of Professor Curtiss is concerned with results which Laguerre deduced from his proof of the applicability of Descartes' rule of signs to infinite series. Recent completions of Laguerre's theorems are especially considered.

10. It appears that the most general solution of the differential equations of motion of an incompressible viscous fluid was obtained by Lamb for the case of steady motion when the inertia terms are neglected. A further solution for the case

*Transactions, vol. 6, No. 3 (July, 1905). 
[Mar.,

of small motion has also been deduced in a general form for spherical surfaces. The paper by Mr. Poor exhibits solutions for the cases of steady and non-steady motion without neglecting the inertia terms. These solutions are obtained by expanding the velocity and the average normal pressure in powers of an arbitrary parameter, thus reducing the problem to the solution of an infinite sequence of integral equations. The second approximation for the translation of a sphere through an infinite fluid at rest at infinity is deduced.

11. In his "Second report on recent progress in the theory of groups of finite order," published in this Bulletrn, volume 9 (1902), Professor Miller stated, on page 116, that " no one seems to have investigated the question whether a nonabelian group can have an abelian group of isomorphisms." In Hilton's Finite Groups, 1908, page 233, the question "Can a non-abelian group have an abelian group of isomorphisms?" is placed in the list of "a few interesting questions still awaiting solution."

In the present paper Professor Miller proves the existence of a non-abelian group of order 64 which actually has an abelian group of isomorphisms. This non-abelian group $G$ can be generated by four operators $s_{1}, s_{2}, s_{3}, s_{4}$ which satisfy the following conditions:

$$
\begin{gathered}
s_{1}^{8}=1, \quad s_{2}^{2}=s_{3}^{2}=s_{4}^{2}=1, \quad s_{2} s_{1} s_{2}=s_{1}^{5}, \quad s_{3} s_{1} s_{3}=s_{1}, \\
s_{4} s_{1} s_{4}=s_{1}, \quad s_{3} s_{2} s_{3}=s_{2}, \quad s_{4} s_{2} s_{4}=s_{3} s_{2}, \quad s_{3} s_{4} s_{3}=s_{4} .
\end{gathered}
$$

The central of $G$ is generated by $s_{1}{ }^{4}$ and $s_{3}$, and the group of inner isomorphisms is of order 8 and of type $(1,1,1)$. The three operators of order 2 in the central of $G$ are invariant under its group of isomorphisms. The latter group is abelian, of order 128, and of type $(1,1,1, \cdots)$.

12. In the Appendix to Hilton's Introduction to the Theory of Groups of Finite Order, 1908, page 233, the following question is asked: "Can a group of order $p^{a}$ have a group of automorphisms whose order is also a power of $p$ ?" Professor Miller proved the existence of a group of order $p^{9}, p$ being any prime number whatever, whose group of isomorphisms has an order which is a power of $p$. This group is generated by three operators $s_{1}, s_{2}$, and $s_{3}$, of orders $p^{4}, p^{3}$, and $p^{2}$ respectively, which satisfy the following conditions: 


$$
s_{1}^{-1} s_{3} s_{1}=s_{1}{ }^{p 3} s_{3}, \quad s_{1}{ }^{-1} s_{2} s_{1}=s_{2}{ }^{p+1}, \quad s_{2}{ }^{-1} s_{3} s_{2}=s_{3}{ }^{p+1} .
$$

It contains two characteristic subgroups of order $p$, generated by $s_{1}$ and $s_{3}$ respectively, and it involves at least one characteristic subgroup of every order which divides the order of the group. Its central is of order $p^{6}$ and the central quotient group is abelian and of type $(1,1,1)$. The central involves the $p$ th power of every operator of the group and it is generated by such operators.

13. In the second edition of Burnside's Theory of Groups of Finite Order, 1911, page 89, it is stated that " no case is known of an outer isomorphism which changes each conjugate set of operations into itself. On the other hand, it is still an open question whether or no such isomorphisms exist." In the present paper Professor Miller proves the existence of a group $G$ of order 128 which admits such isomorphisms. This group may be generated by seven operators of order 2, which will be denoted by the symbols $t_{1}, t_{2}, t_{3}, s_{1}, s_{2}, s_{3}, s_{4}$.

The first three of these operators generate the central of $G$, which is of order 8. The properties of the other operators are defined by the following equations:

$$
\begin{aligned}
& s_{1} s_{2} s_{1}=t_{1} s_{2}, \quad s_{1} s_{3} s_{1}=t_{2} s_{3}, \quad s_{1} s_{4} s_{1}=t_{3} s_{4}, \\
& s_{2} s_{3} s_{2}=t_{2} t_{3} s_{3}, \quad s_{2} s_{4} s_{2}=t_{2} s_{4}, \quad s_{3} s_{4} s_{3}=s_{4} .
\end{aligned}
$$

To prove that this group has an outer isomorphism which changes each set of conjugate operators into itself, it may be observed that the isomorphism which transforms $s_{4}$ into $t_{3} s_{4}$, and transforms each of the three operators $s_{1}, s_{2}, s_{3}$ into itself, is an outer isomorphism of $G$. On the other hand, it can be easily proved that this outer isomorphism transforms into itself each set of conjugate operators of $G$. See W. Burnside, Proceedings of the London Mathematical Society, volume 11 (1912), page 40 .

14. The problem of the deviations of falling bodies was taken up by Professor F. R. Moulton because of the divergence of results which have been secured by different methods. A body dropped from a point $P_{0}$ near the surface of a rotating mass $M$, and moving subject only to the attraction of $M$, strikes its surface at $P_{1}$. A plumb-line suspended from $P_{0}$ touches the surface of $M$ at $P_{2}$. The deviations of 
[Mar.,

the falling body are the distances of $P_{1}$ from $P_{2}$ expressed either in geocentric angular measure, or linear measure with the distances measured along the surface of the earth. It is shown how to locate $P_{1}$ and $P_{2}$ for any potential function. Application is made to the case where the potential function is expansible in zonal harmonics, and it is found that the meridional deviation is composed of two parts, one away from the equator and one toward the equator, of which the latter is numerically the more important.

15. At the summer meeting of the Society at Madison, Professor Wilczynski announced the result that the finite equations of surfaces whose directrix curves are indeterminate and whose asymptotic curves belong to linear complexes can be obtained by quadratures. He now finds that the equations of these surfaces, referred to their asymptotic lines, may be written in the still simpler form

$$
\begin{aligned}
& x=-2\left(U^{\prime}-V^{\prime}\right)(u-v)+4(U+V), \\
& y=U^{\prime}-V^{\prime}, \quad z=u+v,
\end{aligned}
$$

where $U$ and $V$ are arbitrary functions of the single variables $u$ and $v$, and where $U^{\prime}$ and $V^{\prime}$ are the derivatives of these functions. To avoid the appearance of ruled surfaces, which are only in an improper sense solutions of the problem, the third derivatives of $U$ and $V$ must not vanish identically.

Equations (1) are related to the earlier equations, given at the summer meeting, in very much the same way as the formulae of Weierstrass for minimal surfaces are connected with those of Enneper.

As in that other theory, the surfaces (1) are algebraic if and only if the functions $U(u)$ and $V(v)$ are algebraic functions of their arguments. In the simplest case, when $U$ and $V$ are polynomials of the third degree, the resulting surface is a rational sextic with twisted cubic curves for asymptotic lines.

16. Professor Love's paper considers the existence and form of asymptotic integrals of the homogeneous linear differential equation of the $n$th order, valid for large positive values of the independent variable. The coefficients are assumed to be developable in asymptotic (or convergent) power series. 
No restriction is laid upon the roots of the characteristic equation as regards their order of multiplicity, but a certain assumption of a different nature is made in order to reduce the algebraic difficulties of the analysis. This assumption is such that the familiar case of distinct roots is included as a special case of the problem treated. It is shown that if the characteristic equation has $l$ distinct roots, the $n$ fundamental solutions of the differential equation fall into $l$ groups, in such a way that corresponding to a $p$-fold root there is a group of $p$ fundamental solutions proceeding in powers of $(1 / x)^{1 / p}$.

17. Professor Shaw shows that the two fundamental operations in vector analysis of $n$ dimensions lead to the progressive products of Grassmann and to products of reduced order, from which two kinds of products, the complete associative productive may be built up and defined. If one of the vectors is the symbolic Hamiltonian $\nabla$, they lead to the extension of the divergence and curl.

18. For the problem of the calculus of variations with fixed end points, the curves which minimize an integral of the form

$$
J=\int F\left(x, y, z, x^{\prime}, y^{\prime}, z^{\prime}\right) d S
$$

have previously been studied, provided that the minimizing arc along which the integral $J$ is taken is free to traverse any portion of space or is restricted to lie entirely within a given region $R$. It may happen, however, that while no extremal joining the two fixed end points exists entirely interior to a region $R$, yet there may be a minimizing curve consisting partly of arcs interior to the region and partly of arcs along its boundary. This gives rise to the problem of investigating the properties which characterize a minimizing curve lying partly on a given surface $S$, while the variations with which it is compared are restricted to lie on one side of the surface, a problem analogous to one in the plane which has been discussed by Professor Bliss. The paper of Professors Bliss and Underhill has to do with the problem of a minimum of the integral above with respect to unilateral variations in space, that is, variations which lie on one side of a given surface. The study of the problem is justified by the fact that the conditions found, especially those analogous to the 
usual Jacobi condition, are not merely generalizations of the corresponding conditions in the plane, but present new characteristics of interest.

19. In a recent paper in the Mathematische Annalen (volume 73 (1913), page 596) Behaghel has deduced, for problems of the calculus of variations in space, an expression for the Weierstrass $E$-function which is analogous to a well-known formula for similar problems in the plane. The result which he obtains is correct, but in his proof he uses functions, in his notation $A, B, C$, which may become infinite even for very simple problems. For such cases his proof would not be valid. In the paper of Professor Bliss an expression of slightly different form is obtained for the $E$-function, which may be reduced to that of Behaghel by a simple transformation, and a discussion is given of some of the properties of the $E$-function resulting from the formula. The methods are applicable to space of any number of dimensions.

20. In this paper the fundamental domain is taken to be the linear continuum between $x=0$ and $x=1$. Every irrational point $c$ in that domain may be represented as a continued fraction in the form

$$
\frac{1}{c_{1}}+\frac{1}{c_{2}}+\frac{1}{c_{3}} \ldots+\frac{1}{c_{n}}+\cdots
$$

where $c_{n}(n=1,2, \cdots, \infty)$ is a positive integer. The theorem proved by Dr. Blumberg is as follows:

I et $(K)$ be any given condition by virtue of which the following functions are determined:

$$
\begin{aligned}
\bar{n} & =F\left(n, c_{1}, c_{2}, \cdots, c_{n}\right), \\
c_{n+1}{ }^{\prime} & =F_{1}\left(n, c_{1}, c_{2}, \cdots, c_{n}\right), \\
c_{n+2} & =F_{2}\left(n, c_{1}, c_{2}, \cdots, c_{n}\right), \\
\cdot & \cdot \cdot \cdot \cdot \cdot \cdot \\
c_{n+\bar{n}^{\prime}} & =F_{\bar{n}}\left(n, c_{1}, c_{2}, \cdots, c_{n}\right) .
\end{aligned}
$$

$F, F_{1}, F_{2}, \cdots, F_{\bar{n}}$ are assumed to be single-valued and to take only positive integral values for the arguments in question. Otherwise these functions are arbitrary. Let $S_{1}$ denote the set of irrational points $c$ for which in the representation (1) it ha ppens only for a finite number of values of $n$ that 


$$
c_{n+1}{ }^{\prime}=c_{n+1}, c_{n+2}{ }^{\prime}=c_{n+2}, \cdots, c_{n+\bar{n}^{\prime}}=c_{n+\bar{n}},
$$

and let $S_{2}$ be the complementary set of irrational points; then $S_{1}$ is of first category and $S_{2}$ of second category.

A special case of the theorem is as follows: The set of points $c$ for which

$$
\frac{1}{c_{1}}+\frac{1}{c_{2}}+\frac{1}{c_{3}}+\cdots
$$

is convergent is of first category; the set of points $c$ for which the series (2) is divergent is of second category.

21. In a paper read at the November, 1913, meeting of the Southwestern Section of the Society, Dr. Blumberg gave certain properties of the oscillation function $\omega f(x)$. By the aid of these results, he is able to solve completely the oscillation equation

$$
\omega \omega f(x)=g(x) \omega f(x),
$$

where $g(x)$ is any given continuous function of $x$ and $f(x)$ is to be determined. The equation

$$
\omega f(x)=g(x) f(x)
$$

may in a certain sense be reduced to an equation of the form (1).

22. The discriminating analysis by Brouwer in the November Bulletin (pages 81-96) puts in evidence the rôle that awaits a non-enumerable well-ordered set obtained without the intervention of the continuum. In this paper Professor Frizell proves that Axioms $T$ in his "Axioms of ordinal magnitudes," presented to the Cambridge Congress, yield a set that is not countable. The method of proof is based upon that used by Cantor in proving the continuum nicht-abzählbar. A corollary is that, unless the set in question possesses the potency of the continuum, we have here a non-enumerable well-ordered set of lower potency than the continuum.

23. In this paper Professor E. J. Moulton considers an application of integral equation theory to the theory of figures of equilibrium of a rotating compressible fluid mass. Certain negative theorems are first proved, which are closely 
related to well-known theorems of Hamy, Volterra, and Vérronet. They allow generality in the surfaces in contrast with the generality of the density functions of the earlier theorems. Figures of equilibrium are next discussed as functions of a compressibility parameter. A new method is thus obtained for arriving at the Poincare "forms of bifurcation." Finally the methods are applied to the determination of the theoretical shape of the earth. By comparing the observed with the theoretical figure it seems certain that the assumed Laplace pressure-density equation is not approximately satisfied in the earth's interior.

24. As a direct generalization of real quaternions, Professor Dickson considers the linear algebra with the $n^{2}$ units $i^{s} j^{t}(s, t=0,1, \cdots, n-1)$ and coordinates in a field $F$, where

$$
\phi(i)=0, \quad j i=\theta(i) j, \quad j^{r}=g,
$$

$g$ and the coefficients of the polynomials $\phi$ and $\theta$ being in $F$. Moreover, let $\phi(x)=0$ be a uniserial abelian equation in $F$, i. e., an equation irreducible in $F$ and having the roots

$$
i, \theta(i), \theta^{2}(i) \equiv \theta[\theta(i)], \cdots, \theta^{r-1}(i) \quad\left[\theta^{r}(i) \equiv i\right] .
$$

This associative algebra is obtained and discussed in the Transactions for January, 1914. For $n=2$, we may take $\phi=x^{2}-c$; then right hand and left hand division except by zero is always possible and unique (briefly, it is a division algebra) if and only if $g$ is not the norm $e^{2}-c f^{2}$ of any number $e+f i$ in the field $F(i) *$ The corresponding theorem for $n=3$ is proved. Soon after the above algebra in $n^{2}$ units was communicated to Professor Wedderburn, he succeeded in proving that, for any value of $n$, it is a division algebra if and only if $g$ is not the norm of any number in $F(i)$. (See abstract 34 on page 301 ).

25. Any number of Cayley's algebrat with the units $1, e_{1}, \cdots, e_{7}$ may be expressed in the form $q+Q e$, where $q$ and $Q$ (also $r$ and $R$ below) are real quaternions in the units $1, e_{1}, e_{2}, e_{3}$, while $e=e_{4}$. By use of the multiplication table

* Transactions, vol. 13 (1912), pp. 65, 66.

$\dagger$ Coll. Math. Papers, I, p. 127; XI, p. 368. 
for Cayley's eight units, it can be verified with the exercise of patience that

$$
(q+Q e)(r+R e)=q r-R^{\prime} Q+\left(R q+Q r^{\prime}\right) e,
$$

where $r^{\prime}, R^{\prime}$ are the quaternions conjugate to $r, R$. Starting with this condensed formula as the complete multiplication table of the algebra, Professor Dickson finds at once that the norm of a product is the product of the norms of the factors $\left(q q^{\prime}+Q Q^{\prime}\right.$ being the norm of $\left.q+Q e\right)$. Also at once that right and left-hand division except by zero is always possible and unique. These proofs rest upon the simplest algebraic properties of quaternions. They are given in $\$ 14$ of Linear Algebras, in press for the Cambridge Tracts.

26. In his first paper Mr. Schweitzer proved the following theorem: If $f\{x, f(y, z)\}=f\{z, f(y, x)\}$ and $\phi\{f(x, y), y\}=\psi(x)$ and $f\{\phi(x, y), x\}=\psi(y)$ and $f\{\psi(x), \psi(y)\}=f(x, y)$, then there exists a function $\chi(x)$ such that $\chi[\psi(x)]=\chi(x)+c$, $\chi[f(x, y)]=\chi(x)-\chi(y)$ and $\chi[\phi(x, y)]=\chi(x)+\chi(y)+c$, where $c$ is a constant. If in particular one assumes $\psi(x)=x$, that is, $c=0$, then this theorem reduces to a theorem previously obtained by the author.

The preceding relations are satisfied by $\psi(x)=\sqrt{x^{2}-1}$, $f(x, y)=\sqrt{x^{2}-y^{2}}, \phi(x, y)=\sqrt{x^{2}+y^{2}-1}$ and accordingly the following problem is investigated: Given the quasitransitive and symmetric functions $f(x, y)$ and $\phi(x, y)$, to determine the functions $\Omega_{f}(x), \Omega_{\phi}(x), \chi_{f}(x), \chi_{\phi}(x)$ such that the relations

$$
\begin{aligned}
& f(x, y)=\Omega_{f}\left\{\chi_{f}(x)+\chi_{f}(y)\right\} \cdot \Omega_{f}\left\{\chi_{f}(x)-\chi_{f}(y)\right\}, \\
& \phi(x, y)=\Omega_{\phi}\left\{\chi_{\phi}(x)+\chi_{\phi}(y)\right\} \cdot \Omega_{\phi}\left\{\chi_{\phi}(x)-\chi_{\phi}(y)\right\}
\end{aligned}
$$

are satisfied. Reference is made to the article of Lelieuvre, Bulletin des Sciences mathématiques, series 2, volume 27, page 31.

27. In the American Journal, April, 1912, it is shown how to construct the systems ${ }^{2} R_{2}{ }^{(0)},{ }^{3} R_{3}{ }^{(0)},{ }^{4} R_{4}{ }^{(0)}$, etc., as projective analogues of the systems ${ }^{1} R_{1},{ }^{2} R_{2},{ }^{3} R_{3}$, etc. It is also shown, l. c., that the systems ${ }^{2} R_{2}$ and ${ }^{2} R_{2}{ }^{(0)}$ may be generalized into a single system, say, ${ }^{2} R_{2}{ }^{(1)}$. Analogously the systems ${ }^{3} R_{3}$ and ${ }^{3} R_{3}{ }^{(0)},{ }^{4} R_{4}$ and ${ }^{4} R_{4}{ }^{(0)}$, etc., may be generalized into systems 
${ }^{3} R_{3}{ }^{(\mathrm{I})},{ }^{4} R_{4}{ }^{(\mathrm{I})}$, etc., respectively. An $n$-dimensional point field Mr. Schweitzer defines to be the set of points satisfying (at least) the system ${ }^{n} R_{n}{ }^{\text {(I) }}(n=2,3, \cdots)$. For example, a two-dimensional point field is given by the set of points in the interior and on the boundary of a circle of finite radius $\neq 0$ in the euclidean plane, since this set satisfies (American Journal, l. c.) the system ${ }^{2} R_{2}{ }^{(1)}$. Similarly, the set of points in the interior and on the boundary of a hypersphere of finite radius $\neq 0$ in euclidean $n$-dimensional space $(n=2,3, \cdots)$ satisfies the system ${ }^{n} R_{n}{ }^{(\text {() }}$. Essentially two categories of point fields are to be distinguished, the sensed and the non-sensed. The latter are easily defined in terms of the former.

Applying the preceding conceptions to certain investigations* of E. Müller, the author constructs an $(n+1)-$ dimensional geometry $(n=2,3, \cdots)$ consisting of (1) the set of all circles (boundary and interior) of finite radius $\neq 0$ in the euclidean plane; (2) the set of all spheres (boundary and interior) of finite radius $\neq 0$ in the euclidean three-space, and so on. In particular, the sets of circles, spheres, etc., of the same $\dagger$ finite radius $\neq 0$ are investigated in each geometry. Two cases are to be distinguished according as a definite sense is or is not attached to the circular, spherical, etc., fields. In the non-sensed case, the set of all circular fields in the euclidean plane, gives rise to a three-dimensional hyperbolic geometry; similarly, the set of all non-sensed spherical fields in euclidean three-space gives rise to a four-dimensional hyperbolic geometry. When circular (spherical) fields are taken of the same radius there arises a limit surface abstractly identical with a euclidean plane (three-space).

Since in the preceding $(n+1)$-dimensional geometry the elements are $n$-dimensional point fields, the geometry may be regarded as a theory on a class of relations $\left\{{ }^{n} R_{n}{ }^{(\mathrm{I})}\right\}$. This is compared with $r$-dimensional geometries constructed on the basis of a class of relations $\left\{r R_{r}\right\}$, where $r<n$.

28. Recent discussion regarding the laws of sliding friction makes problems in constrained motion of special interest. Professor Field considers the motion of two particles which are connected by a rigid weightless rod, one or both of the

* E. Müller, Dissertation (Königsberg), where further references will be found.

† Cf. Müller, 1. c., p. 279, (c). 
particles being constrained to move on a rough curve or surface. The paper is devoted to a study of what the initial conditions must be in order that the equations of motion may give rise to $(a)$ conditions which are compatible, (b) unique values for the accelerations involved.

29. The purpose of Dr. Williams's paper is to obtain a simple solution of the equation

$$
f(x+1)=E(x) f(x),
$$

where $E(x)$ is an entire or a meromorphic function. The method employed makes use of the Weierstrass factor form of $E(x)$. Barnes has also given a treatment of the equation by making use of the factor form of $E(x)$. His solution, however, contains certain complicated functions of the zeros and poles of $E(x)$, for which he was able to obtain the explicit form only in special cases. Dr. Williams proceeds in a way different from that used by Barnes, and obtains a solution which is completely determined. The method is also simpler, and exhibits in a clear way why certain convergence factors can be easily obtained.

30. In this paper Dr. Sullivan discusses a net of directrix curves which are related to a ruled surface in much the same way that the directrix curves introduced by Professor Wilczynski* are related to a curved surface. These curves and their associated linear congruences are completely determined by certain fundamental invariants $h, \theta^{\prime}, C^{\prime}$. When the twisted asymptotic curves belong to linear complexes the directrix curves form a conjugate system, and they can be found by quadratures. Theorems analogous to those on pages $98,118,119,120$ of the second memoir have been found for ruled surfaces. The directrix curves are indeterminate and the corresponding congruences degenerate, if and only if the invariants $h, \theta^{\prime}, C^{\prime}$ vanish, that is, if and only if the surface is a Cayley cubic scroll. In this case the directrix congruences degenerate into the net of lines in the uniplane, and the sheaf of lines through the unode of the surface.

31. The first part of the paper by Dr. Wahlin is a development of some general properties of relative $p$-adic domains,

* Second memoir, Transactions, vol. 9 (1908). 
and equations in an arbitrary $p$-adic domain. The main theme of the paper is, however, the determination of the nature of the $p$-adic domains in which the binomial equation of degree $l, l$ being a prime, has $l$ solutions. The equation is considered for the domains of the various prime divisors of an arbitrary algebraic domain, and in each case it is determined what the simplest irrationality is which must be adjoined to the given domain, in order that the equation in question may have $l$ solutions.

32. In this paper Professor Bauer and Dr. Slobin investigate the relation of the argument of a trigonometric function to the function from the standpoint of the nature of the number represented. They set up various forms of the argument for which the function always represents algebraic numbers, and other forms for which the function represents transcendental numbers. It is shown that the trigonometric functions represent algebraic numbers if the argument is a rational multiple of $\pi$. A symbolic method is indicated whereby the algebraic equation may be set up for roots of the indicated type. The following is representative of the theorems developed: If a trigonometric function represents a number of the form $a \pi$, where $a$ is an algebraic number other than zero, the argument is of the form $\log t \pi$, where $t$ is a transcendental number.

33. The definition of the sum of a double series given by Pringsheim admits of conditionally convergent double series, while the definition of convergence given by Jordan does not. Adopting Pringsheim's definition, Professor Bauer shows that in a conditionally convergent double series

$$
\lim _{p=\infty, q=\infty} S_{p q}=\lim _{m=\infty} S_{m m} .
$$

This leads to a definition of multiplication for single series whereby two conditionally convergent series may be multiplied together with the assurance that the limiting sum of the resulting series will be equal to the product of the limiting sums of the given series. It is also shown that a conditionally convergent double series whose terms tend to zero may be rearranged so that the limiting sum of the rearranged double series is equal to any preassigned quantity. 
34. This paper is a more general treatment of the subject presented to the Society by Professor Bates in April, 1910. In the former paper, only one value of $\lambda$ (namely, $n-1$ ) was considered.

H. E. Slaught, Secretary of the Chicago Section.

\section{SHORTER NOTICES.}

Leçons sur le Prolongement analytique. By Ludovic ZoRetri. Paris, Gauthier-Villars, 1911 . vi+115 pp.

IT is the plan of this little volume, like that of its fellows of Borel's excellent collection of monographs on the theory of functions, to conduct a student from a presupposed minimum of knowledge-but hardly of capacity - to the actual present frontier of science. This is evidenced by the many questions raised, but not to-day answerable, to be found scattered through its pages. It consists, in substance, of lectures delivered by its author at the Collège de France during the year 1908-1909; while it may be said to have for its subject such questions of the theory of functions as can be best treated, not by the methods of Cauchy and Riemann, but by going back to Weierstrass's fundamental definition of an analytic function. It is in this sense that the title is to be understood. Weierstrass's definition gives rise, in the general case, to a function with an infinite number of branches. The small advance that has been made in the study of such functions, the importance of which is incontestable (in the analytic theory of differential equations, for instance), is due, in part, to its difficulty, in part to the lack of appropriate tools. In particular-it is our author's idea-progress in the theory of these functions depends on progress in the theory of the Riemann's surface of an infinite number of sheets (as this latter is conceived of by Poincare in his paper on the uniformization of functions, Acta Mathematica, volume 31, 1907). " The object of this book will have been accomplished if I succeed in persuading the reader of the great interest attaching to investigations of these surfaces" (page 43).

The work begins, after an interesting and suggestive introduction of six pages, with a chapter of 22 pages on (closed) 